\title{
HLA-B27-induced ER stress might promote bone loss in SpA
}

HLA-B27 is strongly associated with spondyloarthritis (SpA) and has been suggested to promote this disease through the aberrant initiation of immune responses. Now, Robert Colbert and colleagues have uncovered an additional pathogenic mechanism involving HLA-B27, which may explain the excess bone loss that occurs in SpA. "Our previous work has shown that HLA-B27 can misfold, and that when this occurs it can cause cell-intrinsic biological effects that are unrelated to its natural function presenting antigenic peptides to T cells," begins Colbert. "We therefore asked whether HLA-B27 misfolding might affect cells involved in bone homeostasis." To investigate, the researchers obtained bone marrow monocytes from HLAB27-transgenic rats and cultured them with TNF, a cytokine that is increased in $\mathrm{SpA}$ and that can induce the differentiation of osteoclasts from bone marrow monocyte populations. They found that in vitro TNF treatment amplified the expression of HLA-B27 by the transgenic monocytes, and this upregulation was associated with evidence of misfolding and endoplasmic reticulum (ER) stress, which was not observed in TNF-treated wild-type monocytes.

But does this ER stress have any impact on osteoclastogenesis? Indeed, compared with wild-type and HLA-B7-transgenic control monocytes, HLA-B27-transgenic monocytes generated higher numbers of osteoclasts in response to TNF. This effect could be reversed by the addition of a blocking antibody specific for IL-1a, the production of which was elevated in the TNF-treated cells. Importantly, treatment of wild-type monocytes with a chemical inducer of ER stress also augmented the expression of IL-1 $\alpha$. These results suggest that, in response to TNF, ER stress resulting from HLA-B27 misfolding enhances the production of IL-1 a by monocytes, which promotes the differentiation of these cells into osteoclasts. In vivo, such an increase in osteoclast numbers would be expected to boost bone resorption. Further experiments showed that the production of the antiosteoclastogenic cytokine IFN- $\beta$ was also upregulated in TNF-treated HLA-B27-transgenic monocytes, but in this in vitro system the effects of IL-1 a predominated.

"These are the first studies that link HLA-B27 to altered bone homeostasis, which may be relevant to the pathogenesis of ankylosing spondylitis," concludes Colbert. He plans to extend his studies to confirm the relevance of the findings in human cells.

Isabel Woodman

Original article Layh-Schmitt, G. et al. HLA-B27 alters the response to TNF $\alpha$ and promotes osteoclastogenesis in bone marrow monocytes from HLA-B27 transgenic rats. Arthritis Rheum. doi:10.1002/art.38001 